\title{
Benchmarks
}

Piotr M. Skowron, EURx Ltd., ul. Jaskowa Dolina 29, 80-286 Gdansk, Poland. Internet: piotrs@gdansk.sprint.pl

Received 9 February 1999; accepted 19 July 1999.

\author{
Sylwia M. Rutkowska ${ }^{1}$ \\ and Piotr M. Skowron" ${ }^{1,2}$ \\ ${ }^{1}$ EURx, Ltd. \\ ${ }^{2}$ Technical University \\ of Gdansk \\ Gdansk, Poland
}

\section{In Vivo Measurement of Biodistribution Kinetics of Radiolabeled Compounds in Laboratory Animals}

BioTechniques 27:934-938 (November 1999)

In pharmacokinetic studies, both the biodistribution and the time course of the distribution are of interest. There are two ways of obtaining kinetic data: (i) laboratory animals are injected with the radiolabeled reagent and sacrificed after certain time points; the radioactivity in each organ is then measured separately. This method requires a number of animals for every single time point; (ii) gamma-camera images are obtained to follow the kinetics in individual mice in vivo, a method that drastically reduces the number of animals needed, as well as the amount of radioactivity used. However, the second way requires expensive radiological equipment, which is not accessible to all laboratory scientists.

Here, we describe a simple and inexpensive method to obtain in vivo kinetic data using equipment that is present in every laboratory. Measurements of gamma and X-rays were taken with a modified radiation monitor. The modification consists of narrowing down the open-window area of the probe to a small slit or dot (see Figure 1). This step allows one to measure radiation emitted from various body regions in animals as small as mice. Because with these measurements the localization is not as exact as with the conventional method of counting separate organs, the proposed method was used in addition, i.e., organs were counted in a scintillation counter at the end of the experiment. In this way, kinetic data was obtained by monitor measurements and exact location was determined by counting separate organs.

A $\mathrm{p} / \mathrm{m}$ scintillation probe of a Minimonitor series 900 (Mini-Instruments LTD, Essex, England, UK), ranging from $15-500 \mathrm{keV}$, and built for detection of $125 \mathrm{I}$, was used. The monitor was modified as follows: a lead shield was built from lead sheets, to slide over the probe and leave an open area of variable size and shape. First, rectangular pieces of 1.5-2 mm-thick lead sheets were cut to a size of $10 \mathrm{~cm} \times 16 \mathrm{~cm}$ and rolled to form a cylinder that would fit snugly over the probe (with a $1 \mathrm{~cm}$ overlap). The cylinder was soldered together with lead solder. The two round end plates were made from the same lead foil, but in two layers $(3-4 \mathrm{~mm}$ thick). A slit of $1.5 \times 15 \mathrm{~mm}$ was cut out in the middle of each plate. One end plate was soldered to the end of the lead cylinder, leaving an open area of $1.5 \times$ $15 \mathrm{~mm}=23 \mathrm{~mm}^{2}$ in the window of the probe. The second round plate was taped over the first one at a variable angle that further regulated the open area. Taping the plate so that the two slits were perpendicular to each other left a square of $1.5 \times 1.5 \mathrm{~mm}$ or $2.3 \mathrm{~mm}^{2}$. Taping the plate at an angle other than $90^{\circ}$ left a bigger open area that was between 2.3 and $23 \mathrm{~mm}^{2}$. This allowed us to adjust the sensitivity in separate animal experiments in which we used different total injected doses. The costs for materials were approximately \$20.

Using the modified probe, we could distinguish eight different body regions in mice. We used ${ }^{125} \mathrm{I}$-labeled reagents and measured their biodistribution in nu/nu mice. A total dose of 40-100 million counts per minute was injected intravenously. Free iodine was determined to be less than $5 \%$ in most cases. We obtained kinetic data of 15 different 125I-labeled compounds in a total of 128 mice and compared the data to numbers obtained using the conventional method of counting separate organs. Blood samples were also taken from the tail vein at time points of monitor measurements for blood clearance determination. This was of importance to appreciate the level of background counts due to radioactivity in the blood. It was also important to always keep the same angle and distance for each measurement, so only one person measured one set of data.

Data sets obtained from the two different methods of measurements were consistent with each other. Figure 2 presents an example in which the biodistribution of ${ }^{125}$ I-labeled Paclitaxel (Molecular Probes, Eugene, OR, USA) was investigated in nu/nu mice. Figure 2A shows measurements at five different time points with the modified minimonitor. Figure 2B illustrates the data from separately counted organs (conventional method) after sacrifice of the respective mice. In Figure 2A, counts per minute measured with the

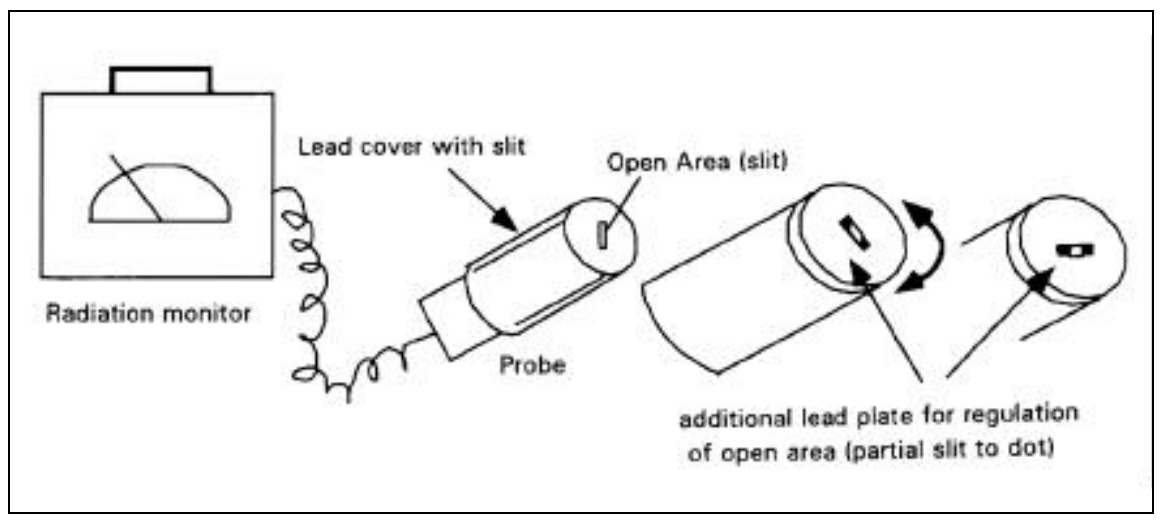

Figure 1. Modified radiation monitor for in vivo measurements of biodistribution kinetics. 


\section{Benchmarks}

modified detector at a distance of 1-2 $\mathrm{mm}$ from the body surface are shown for 8 different body regions: head (brain), cervical area (thyroid), left and right chest region (heart and lungs), left and right upper abdomen (spleen, stomach, liver, colon and intestine) and left and right lower abdominal regions (kidney, bladder, colon and intestine). In the head region, there was a steady

A

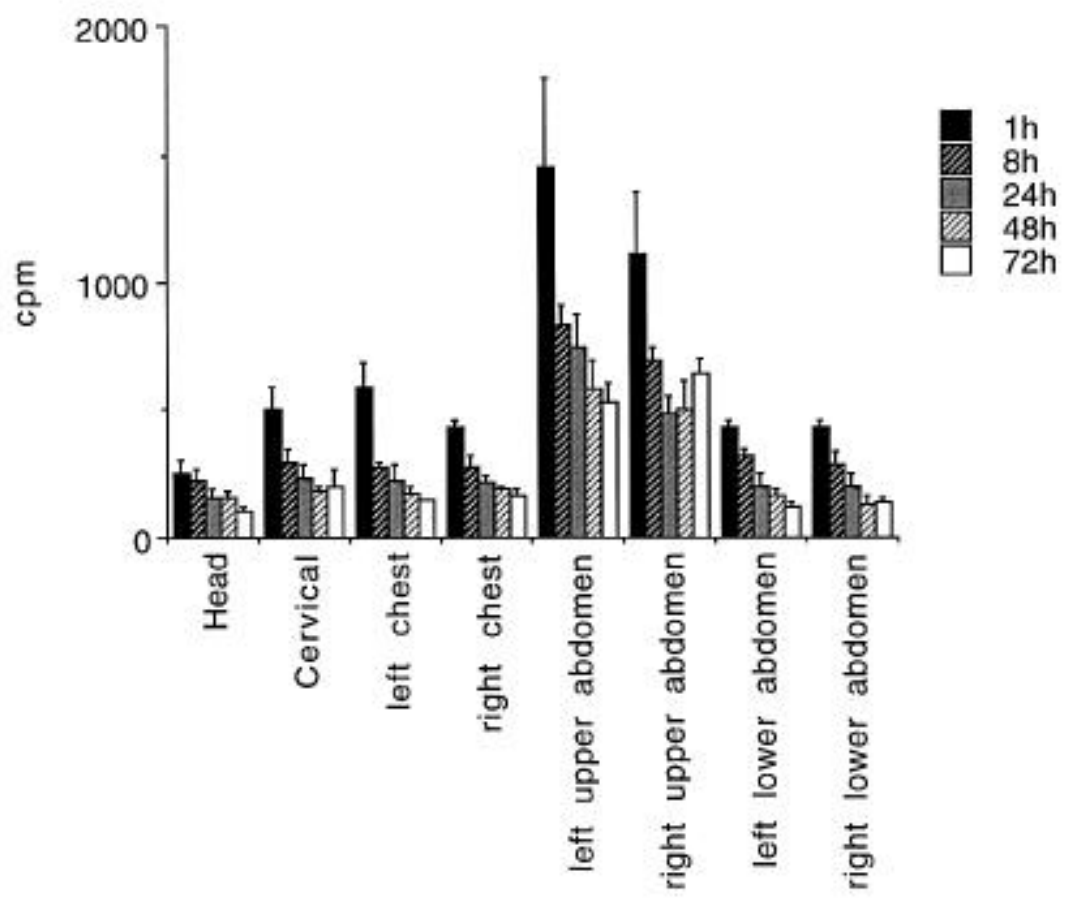

B

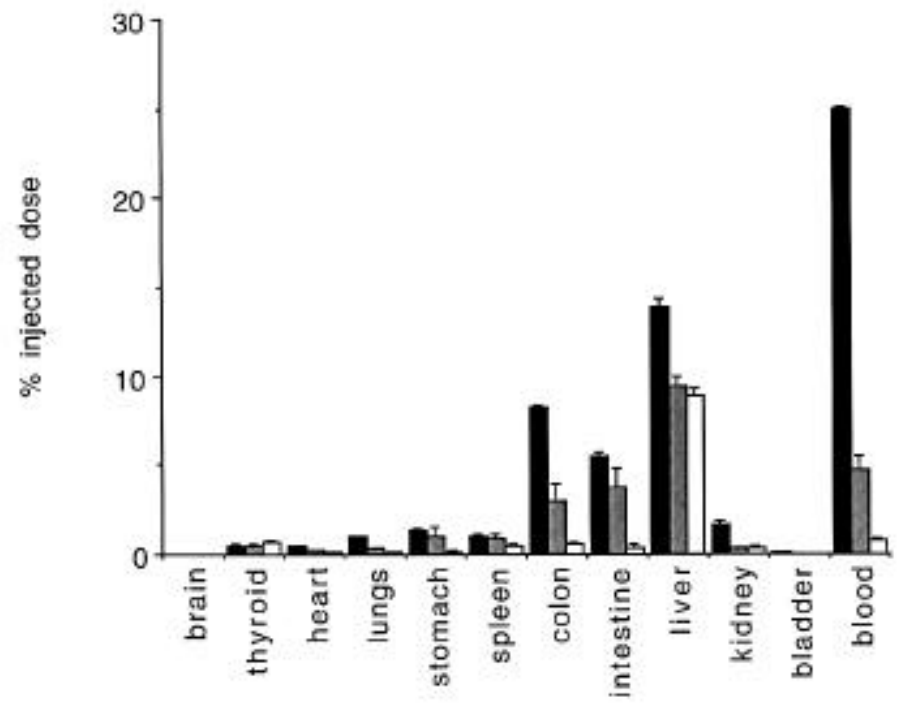

Figure 2. Biodistribution data of ${ }^{125}$ I-labeled paclitaxel in tumor bearing mice obtained with two different methods. (A) In vivo measurement of radioactivity with modified radiation monitor. (B) Radioactivity of separate organs measured in a gamma-counter. 
decrease in counts per minute consistent with decreasing background counts arising from the blood circulation. Taken together with the information in Figure $2 \mathrm{~B}$ that shows no activity in the brain, counts from this region can be interpreted as the level of background activity arising from blood counts. There are only two body regions that show significantly higher radioactive emissions than background-left and right upper abdomen. Organs in this area are stomach (left), spleen (left), colon and intestine (left and right) and liver (mainly right). As already pointed out, the exact origin of the counts has to be determined with the conventional method. In Figure 2B, one can see high counts measured at $24 \mathrm{~h}$ in the organs stomach, spleen, colon, intestine and liver and at $96 \mathrm{~h}$ predominantly in the liver. This is consistent with the high counts seen in the left and right upper abdomen at $24 \mathrm{~h}$ and mainly in the right upper abdomen at $72 \mathrm{~h}$. We learn from Figure 2A that the typical distribution of counts starts very early, that after 24 $\mathrm{h}$ the number of counts in the liver starts to stabilize or accumulate, and that from time point $72 \mathrm{~h}$ on, the predominant activity comes from the liver. At the end point of the experiment, the exact distribution was determined by counting separate organs after sacrificing the mice. The data shown in Figure $2 \mathrm{~A}$ was obtained from three mice. It would have taken 15 mice to get kinetic information from five time points with the conventional method.

The information obtained with the modified monitor is less accurate because gamma and X-rays from more than one organ can hit the probe at the same time, mainly because of the anatomical overlap of organs and to a small extent, counts from adjacent tissue. The amount of the latter can be determined experimentally by measuring a point radiation source and comparing the size of the measured area to the actual known size. In our experiments, the inaccuracy of the detector because of counts from adjacent tissue was not significant in comparison to organ overlap. However, the actual field of view can be decreased, if necessary, by choosing thicker end plates or smaller opening areas, which then might have to be compensated for with a higher injected dose. A second disadvantage of the proposed method is that high blood counts will result in background radiation in organs that are otherwise negative. Therefore, the described method does not replace the conventional method, but brings additional information such as: ( $i$ ) first occurence in time and kinetic behavior of certain biodistribution events that are quantified exactly by the conventional measurement of separate organs; (ii) the definition of the most interesting time points for conventional measurements to answer specific questions; (iii) multiple measurements on the same animal; and (iv) easy recognition of animals that behave atypically. The number of measurements that can be taken is unlimited. Mice tolerate the procedure very well and need no anesthesia.

Multiple measurements in the same animal have other advantages. In stud- ies where tumors are induced by the experimenter, none will ever be exactly the same in two animals. Kinetic data from different animals is therefore only interpretable with caution because differences in radioactive emissions may be due to both the time course and the heterogeneity of the tumors in different mice. In these special cases, the proposed method may even give better information than the conventional one.

In summary, a modification of a standard radiation monitor is introduced, which can be performed at virtually no cost and enables the investigator to obtain additional pharmacokinetic data without using more laboratory mice. Kinetic data is measured with the monitor, and exact localization is determined by counting separate organs at the end of the experiment. Combining the two methods provides a good tool to obtain extensive information with mimimized numbers of research animals. Reducing the number of animals also decreases the amount of radiactive isotopes that have to be handled.

This work was performed at the University of Texas Southwestern Medical Center at Dallas in the laboratory of Dr. P.E. Thorpe. C.G. was supported by a grant of the Deutsche Forschungsgemeinschaft, Az Go 706/1-1. We gratefully acknowledge the assistance of S. Li, E. Lummus and T. Furlough. Animal experiments were performed according to institutional and National Institutes of Health guidelines. Address correspondence to Dr. Claudia Gottstein, University Cologne, Medical Clinics I, LFI E4 R707, Joseph Stelzmannstr. 9, 50924 Cologne, Germany. Internet: claudia. gottstein@uni-koeln.de

Received 16 November 1998; accepted 21 July 1999.

\section{Claudia Gottstein and Richard Forde ${ }^{1}$ \\ University of Texas Southwestern Medical Center at Dallas \\ ${ }^{1}$ Aqua Research \\ Dallas, TX, USA}

